\title{
An Augmented Lagrangian Uzawa Iterative Method for Solving Double Saddle-Point Systems with Semidefinite $(2,2)$ Block and its Application to DLM/FD Method for Elliptic Interface Problems
}

\author{
Cheng Wang ${ }^{1}$ and Pengtao Sun ${ }^{2, *}$ \\ 1 School of Mathematical Sciences, Tongji University, Shanghai 200092, China. \\ 2 Department of Mathematical Sciences, University of Nevada Las Vegas, \\ 4505 Maryland Parkway, Las Vegas, NV 89154, USA.
}

Received 9 May 2020; Accepted (in revised version) 8 October 2020

\begin{abstract}
In this paper, an augmented Lagrangian Uzawa iterative method is developed and analyzed for solving a class of double saddle-point systems with semidefinite $(2,2)$ block. Convergence of the iterative method is proved under the assumption that the double saddle-point problem exists a unique solution. An application of the iterative method to the double saddle-point systems arising from the distributed Lagrange multiplier/fictitious domain (DLM/FD) finite element method for solving elliptic interface problems is also presented, in which the existence and uniqueness of the double saddle-point system is guaranteed by the analysis of the DLM/FD finite element method. Numerical experiments are conducted to validate the theoretical results and to study the performance of the proposed iterative method.
\end{abstract}

AMS subject classifications: 65F10, 65F50, 65N30, 65N85

Key words: Double saddle-point problem, augmented Lagrangian Uzawa method, elliptic interface problem, distributed Lagrange multiplier/fictitious domain (DLM/FD) method.

\section{Introduction}

In this paper, we study a type of augmented Lagrangian Uzawa iterative method for solving a large-scale sparse linear algebraic system as shown below

$$
\mathcal{A} \boldsymbol{u} \equiv\left(\begin{array}{ccc}
A & 0 & C^{T} \\
0 & A_{2} & B^{T} \\
C & B & 0
\end{array}\right)\left(\begin{array}{c}
u \\
u_{2} \\
\lambda
\end{array}\right)=\left(\begin{array}{c}
F \\
G \\
0
\end{array}\right) \equiv \boldsymbol{b},
$$

${ }^{*}$ Corresponding author. Email addresses: wangcheng@tongji.edu.cn (C. Wang), pengtao.sun@unlv.edu (P. Sun) 
where, $\mathcal{A} \in \mathbb{R}^{(n+2 m) \times(n+2 m)}$ is the coefficient matrix, and the right hand side $\boldsymbol{b} \in \mathbb{R}^{n+2 m}$. Inside the coefficient matrix $\mathcal{A}$, the block $A \in \mathbb{R}^{n \times n}$ is symmetric positive definite (SPD), $A_{2} \in \mathbb{R}^{m \times m}$ is symmetric positive semidefinite (SPS), $B \in \mathbb{R}^{m \times m}$ is invertible and $C \in \mathbb{R}^{m \times n}$ with $n>m$. Such linear systems can be derived from the DLM/FD finite element discretization for elliptic interface problems [1,7] and parabolic interface problems [24], where the distributed Lagrange multiplier is employed and acts as a source term for both unknown quantities $u$ and $u_{2}$ in two overlapping domains. We remark that similar, and even much more complicated, multiple saddle-point systems can also be generated from the DLM/FD finite element method for Stokes- [16, 17], Stokes/elliptic- [22] and Stokes/parabolic [23] interface problems, moreover, for fluid-structure interaction (FSI) problems $[11,12,28]$.

Generally, the linear system (1.1) can be viewed as a standard saddle-point system, if we split its coefficient matrix as the following $2 \times 2$ block matrix

$$
\mathcal{A}=\left(\begin{array}{cc|c}
A & 0 & C^{T} \\
0 & A_{2} & B^{T} \\
\hline C & B & 0
\end{array}\right) .
$$

Among the iterative method for solving the saddle-point systems, the Uzawa method, augmented Lagrangian method and their variants are very popular and widely used, due to their simplicity, the minimum requirement of computer memory and the parallel efficiency on emerging multicore and hybrid architectures. The reader are referred to $[2,3,6,8-10,29,30]$ and the references therein. In most of these papers, the convergence analysis are performed under the assumption that the upper left $(1,1)$-block of $\mathcal{A}$ in (1.2) is invertible. However, since the block $A_{2}$ in (1.1) is only positive semidefinite, this assumption is not satisfied. Thus the theocratical results therein cannot guarantee the convergence of these Uzawa type iterative methods for solving the linear system (1.1). Note that in [19] an augmented Lagrangian method has be used for solving the saddle-point system with singular or semidefinite upper left (1,1)-block of $\mathcal{A}$ in (1.2), but the convergence analysis was not given.

On the other hand, we can also split the coefficient matrix $\mathcal{A}$ as another $2 \times 2$ block matrix

$$
\mathcal{A}=\left(\begin{array}{c|cc}
A & 0 & C^{T} \\
\hline 0 & A_{2} & B^{T} \\
C & B & 0
\end{array}\right) .
$$

Since the lower right (2,2)-block in (1.3) itself also owns a saddle-point structure, the linear algebraic system (1.1) is thus treated as a class of double saddle-point system, and fits the definition of a multiple saddle-point operator as given in [21]. Recently, there have been several literatures on the iterative method for solving such three-by-three block systems where the double saddle-point structure, instead of the single saddle-point structure, is studied and used for the construction of iterative method in order to reduce the 
overall workload of the iteration. In [18], some robust preconditioners are constructed for solving the double saddle-point systems, arising from PDE-constrained optimization problems, with the coefficient matrix

$$
\mathcal{A}_{\alpha}=\left(\begin{array}{ccc}
\alpha \breve{M} & 0 & \breve{M} \\
0 & \breve{M} \partial & \breve{A}^{T} \\
\breve{M} & \breve{A} & 0
\end{array}\right),
$$

where $\breve{M}$ and $\breve{M}$ are mass matrices, $\breve{A}$ is from the discretization of the operator $(1-\Delta)$, and $\alpha$ is the regularization parameter. In $[4,5]$, some iterative methods are presented and analyzed for solving double saddle-point problems, of which the coefficient matrix satisfies the following form

$$
\mathcal{B}=\left(\begin{array}{ccc}
\widetilde{A} & \widetilde{B}^{T} & \widetilde{C}^{T} \\
\widetilde{B} & 0 & 0 \\
\widetilde{C} & 0 & -\widetilde{D}
\end{array}\right),
$$

where $\widetilde{A} \in \mathbb{R}^{n \times n}$ is SPD, $\widetilde{D} \in \mathbb{R}^{p \times p}$ is SPS and possibly zero, $\widetilde{B} \in \mathbb{R}^{m \times n}, \widetilde{C} \in \mathbb{R}^{p \times n}$ and $n \geq m+$ $p$. In [15], an alternating positive semidefinite splitting iteration method is proved to be convergent unconditionally for solving the double saddle-point problem with coefficient matrix $\mathcal{B}$ in (1.5) where $\widetilde{A}$ and $\widetilde{D}$ are required to be SPD. It can easily be seen that the matrices $\mathcal{A}_{\alpha}$ and $\mathcal{B}$ are different from the coefficient matrix $\mathcal{A}$ in (1.1), and cannot be turned into $\mathcal{A}$ by means of symmetric permutations (row and column interchanges). We note that the Uzawa type iterative methods have been also applied to double saddlepoint problems in $[13,25]$, where the diagonal block is required to be positive definite, and, the existence and uniqueness of the solution to the double saddle-point problem can be easily obtained from the SPD and full row rank properties of the blocks of its coefficient matrix. Therefore the convergence results of the iterative methods proved in these papers cannot be applied to the case that the double saddle-point problem (1.1) is involved.

The purpose of this paper is to present and analyze an augmented Lagrangian Uzawa method for the double saddle-point problem (1.1), which is our first attempt for the development of an efficient and robust Uzawa type iterative method for solving the multiple saddle point problem derived from the DLM/FD finite element method in a large-scale and long-term simulation of FSI problems. For solving the FSI problems and interface problems, there are many other body-unfitted or body-fitted mesh methods. We note that the linear systems generated by these methods might not be of multiple saddle-point structure, and some iterative algorithms have been developed for solving imperfect interface problems [27] and elliptic interface optimal control problems [14,26], where some other types of body-unfitted mesh methods, instead of DLM/FD finite element methods, are used as the discrete approach.

The rest of this paper is organized as follows: In Section 2, we derive the augmented Lagrangian Uzawa method for solving the problem (1.1), and prove its convergence. In 
Section 3, we briefly recall the DLM/FD finite element discretization for solving the elliptic interface problem, which yields a double saddle-point problem of the form (1.1), and then conduct some numerical experiments to show the performance of the proposed augmented Uzawa method. Finally, we give some conclusions in Section 4.

\section{Augmented Lagrangian Uzawa method for the double saddle-point system}

\subsection{Algorithm Description}

Let $\rho$ and $\omega$ be two positive real numbers, $I$ be the unit matrix, and further let

$$
A_{\rho}=A+\rho C^{T} C, A_{2, \rho}=A_{2}+\rho B^{T} B .
$$

Since $\rho>0$ and $B$ is invertible, we have that $A_{\rho}$ and $A_{2, \rho}$ are SPD.

Define

$$
\mathcal{A}_{\rho}=\left(\begin{array}{ccc}
A_{\rho} & \rho C^{T} B & C^{T} \\
\rho B^{T} C & A_{2, \rho} & B^{T} \\
-\omega C & -\omega B & 0
\end{array}\right)
$$

Then the linear system (1.1) is equivalent to the following system

$$
\mathcal{A}_{\rho} \boldsymbol{u}=\boldsymbol{b} .
$$

Split $\mathcal{A}_{\rho}$ as

$$
\mathcal{A}_{\rho}=\mathcal{A}_{\rho}^{(1)}-\mathcal{A}_{\rho}^{(2)}
$$

where

$$
\mathcal{A}_{\rho}^{(1)}=\left(\begin{array}{ccc}
A_{\rho} & 0 & 0 \\
0 & A_{2, \rho} & 0 \\
-\omega C & -\omega B & I
\end{array}\right), \quad \mathcal{A}_{\rho}^{(2)}=\left(\begin{array}{ccc}
0 & -\rho C^{T} B & -C^{T} \\
-\rho B^{T} C & 0 & -B^{T} \\
0 & 0 & I
\end{array}\right) .
$$

It is easy to check that $\mathcal{A}_{\rho}^{(1)}$ is invertible, and

$$
\left(\mathcal{A}_{\rho}^{(1)}\right)^{-1}=\left(\begin{array}{ccc}
A_{\rho}^{-1} & 0 & 0 \\
0 & A_{2, \rho}^{-1} & 0 \\
\omega C A_{\rho}^{-1} & \omega B A_{2, \rho}^{-1} & I
\end{array}\right)
$$

Then, an augmented Lagrangian Uzawa iterative method for solving (1.1) can be defined as

$$
\boldsymbol{u}^{(n+1)}=\left(A_{\rho}^{(1)}\right)^{-1} \mathcal{A}_{\rho}^{(2)} \boldsymbol{u}^{(n)}+\left(A_{\rho}^{(1)}\right)^{-1} \boldsymbol{b} .
$$


Some simple calculations lead to

$$
\left(\begin{array}{c}
u^{(n+1)} \\
u_{2}^{(n+1)} \\
\lambda^{(n+1)}
\end{array}\right)=M\left(\begin{array}{c}
u^{(n)} \\
u_{2}^{(n)} \\
\lambda^{(n)}
\end{array}\right)+\left(\begin{array}{c}
A_{\rho}^{-1} F \\
A_{2, \rho}^{-1} G \\
\omega\left(C A_{\rho}^{-1} F+B A_{2, \rho}^{-1} G\right)
\end{array}\right)
$$

where

$$
\begin{aligned}
M & =\left(A_{\rho}^{(1)}\right)^{-1} \mathcal{A}_{\rho}^{(2)} \\
& =\left(\begin{array}{ccc}
0 & -\rho A_{\rho}^{-1} C^{T} B & -A_{\rho}^{-1} C^{T} \\
-\rho A_{2, \rho}^{-1} B^{T} C & 0 & -A_{2, \rho}^{-1} B^{T} \\
-\rho \omega B A_{2, \rho}^{-1} B^{T} C & -\rho \omega C A_{\rho}^{-1} C^{T} B & I-\omega C A_{\rho}^{-1} C^{T}-\omega B A_{2, \rho}^{-1} B^{T}
\end{array}\right) .
\end{aligned}
$$

In the following Algorithm 1, the augmented Lagrangian Uzawa method for solving the system (1.1) is described.

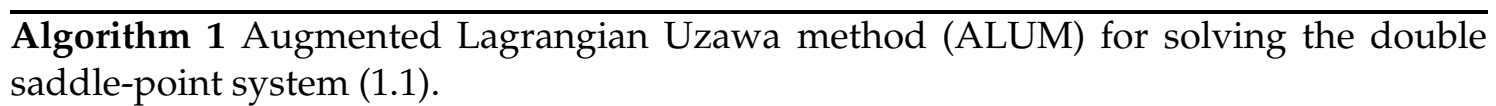

Initialize: $u^{(0)}, u_{2}^{(0)}, \lambda^{(0)}$;

Compute $A_{\rho}$ and $A_{2, \rho}$ by using (2.1);

Repeat

Compute $u^{(n+1)}, u_{2}^{(n+1)}, \lambda^{(n+1)}$ by

$$
\begin{aligned}
& u^{(n+1)}=A_{\rho}^{-1}\left(F-C^{T} \lambda^{(n)}-\rho C^{T} B u_{2}^{(n)}\right), \\
& u_{2}^{(n+1)}=A_{2, \rho}^{-1}\left(G-B^{T} \lambda^{(n)}-\rho B^{T} C u^{(n)}\right), \\
& \lambda^{(n+1)}=\lambda^{(n)}+\omega\left(C u^{(n+1)}+B u_{2}^{(n+1)}\right) ;
\end{aligned}
$$

\section{Until convergence.}

\subsection{Convergence analysis}

To analyze the convergence of the developed iterative method at above, we assume that the double saddle-point problem (1.1) exists an unique solution throughout this subsection. In practice, for instance, the well-posedness of (1.1) that arises from the DLM/FD finite element method has been proved for various interface problems such as the elliptictype [1,7], the parabolic-type [24], the Stokes-type [16, 17], the Stokes/elliptic-type [22] and the Stokes/parabolic-type [23]. 
First of all, we introduce $\mu$ to be an eigenvalue of $M$ with corresponding eigenvector $\left(\tilde{u}^{T}, \tilde{v}^{T}, \tilde{\lambda}^{T}\right)^{T}$, that is,

$$
\begin{aligned}
& -\rho A_{\rho}^{-1} C^{T} B \tilde{v}-A_{\rho}^{-1} C^{T} \tilde{\lambda}=\mu \tilde{u}, \\
& -\rho A_{2, \rho}^{-1} B^{T} C \tilde{u}-A_{2, \rho}^{-1} B^{T} \tilde{\lambda}=\mu \tilde{v}, \\
& -\rho \omega B A_{2, \rho}^{-1} B^{T} C \tilde{u}-\rho \omega C A_{\rho}^{-1} C^{T} B \tilde{v}+\left(I-\omega C A_{\rho}^{-1} C^{T}-\omega B A_{2, \rho}^{-1} B^{T}\right) \tilde{\lambda}=\mu \tilde{\lambda} .
\end{aligned}
$$

Note that the eigenvalue $\mu$ and the eigenvector $\left(\tilde{u}^{T}, \tilde{v}^{T}, \tilde{\lambda}^{T}\right)^{T}$ are complex generally. In order to prove the convergence of the proposed augmented Lagrangian Uzawa method, we only need to prove the spectral radius $\rho(M)$ is less than unity, or to prove each eigenvalue of $M$ satisfying $|\mu|<1$. To that end, we first present some lemmas.

Lemma 2.1. Assume that $\mu$ is an eigenvalue of $M$ with corresponding eigenvector $\left(\tilde{u}^{T}, \tilde{v}^{T}, \tilde{\lambda}^{T}\right)^{T}$. Then $\mu \neq 1$.

Proof. Substituting (2.13) and (2.14) into (2.15), we have

$$
(1-\mu) \tilde{\lambda}=-\mu \omega(C \tilde{u}+B \tilde{v}) .
$$

Assume that $\mu=1$. Then from (2.13), (2.14) and (2.16), we obtain

$$
\begin{aligned}
A_{\rho} \tilde{u}+\rho C^{T} B \tilde{v}+C^{T} \tilde{\lambda} & =0, \\
\rho B^{T} C \tilde{u}+A_{2, \rho} \tilde{v}+B^{T} \tilde{\lambda} & =0, \\
-\omega C \tilde{u}-\omega B \tilde{v} & =0,
\end{aligned}
$$

which imply that the homogeneous linear system $\mathcal{A}_{\rho} \boldsymbol{u}=0$ has a non-zero solution, contradicting with the fact that (1.1), or equivalently (2.3), has an unique solution.

Lemma 2.2. Assume that $\mu$ is an eigenvalue of $M$ with corresponding eigenvector $\left(\tilde{u}^{T}, \tilde{v}^{T}, \tilde{\lambda}^{T}\right)^{T}$. Then $\left(\tilde{u}^{T}, \tilde{v}^{T}\right)^{T}$ is not a zero vector.

Proof. We assume that $\tilde{u}=0$ and $\tilde{v}=0$. It follows from (2.16) that

$$
(1-\mu) \tilde{\lambda}=0 .
$$

Thanks to Lemma 2.1, we have

$$
\tilde{\lambda}=0,
$$

which contradicts with the fact that $\left(\tilde{u}^{T}, \tilde{v}^{T}, \tilde{\lambda}^{T}\right)^{T}$ is an eigenvector.

The following lemma was proved in Theorem 6.2 of [20]. 
Lemma 2.3. [20] Let $\mu$ be the root of the real quadratic polynomial $f(z)=z^{2}+p z+q$. If

$$
1+|p|+q>0, \quad 1-q>0,
$$

then $|\mu|<1$.

Let $(\cdot, \cdot)$ be the inner product of two complex column vectors, that is,

$$
(x, y)=x^{H} y,
$$

where $x^{H}:=\bar{x}^{T}$ denotes the conjugate transpose of $x$.

Now, we are ready to prove the convergence of the developed augmented Lagrangian Uzawa method for solving (1.1).

Lemma 2.4. Assume that $\mu$ is an eigenvalue of $M$ with corresponding eigenvector $\left(\tilde{u}^{T}, \tilde{v}^{T}, \tilde{\lambda}^{T}\right)^{T}$. If $C \tilde{u}+B \tilde{v} \neq 0$, then $|\mu|<1$.

Proof. Using (2.16) in (2.13) and (2.14), we obtain

$$
\begin{aligned}
& -(1-\mu) \rho C^{T} B \tilde{v}+\mu \omega C^{T}(C \tilde{u}+B \tilde{v})=(1-\mu) \mu A_{\rho} \tilde{u}, \\
& -(1-\mu) \rho B^{T} C \tilde{u}+\mu \omega B^{T}(C \tilde{u}+B \tilde{v})=(1-\mu) \mu A_{2, \rho} \tilde{v} .
\end{aligned}
$$

Thus, we have

$$
\begin{aligned}
(\mu-1) \rho(C \tilde{u}, B \tilde{v})+\mu \omega(C \tilde{u}, C \tilde{u}+B \tilde{v}) & =(1-\mu) \mu\left(A_{\rho} \tilde{u}, \tilde{u}\right) \\
(\mu-1) \rho(B \tilde{v}, C \tilde{u})+\mu \omega(B \tilde{v}, C \tilde{u}+B \tilde{v}) & =(1-\mu) \mu\left(A_{2, \rho} \tilde{v}, \tilde{v}\right) .
\end{aligned}
$$

By summing (2.26) and (2.27), we have

$$
\begin{aligned}
& (\mu-1) \rho((C \tilde{u}, B \tilde{v})+(B \tilde{v}, C \tilde{u}))+\mu \omega(C \tilde{u}+B \tilde{v}, C \tilde{u}+B \tilde{v}) \\
= & (1-\mu) \mu\left(\left(A_{\rho} \tilde{u}, \tilde{u}\right)+\left(A_{2, \rho} \tilde{v}, \tilde{v}\right)\right),
\end{aligned}
$$

which implies that $\mu$ is a root of the quadratic polynomial

$$
\mu^{2}+p \mu+q
$$

with coefficients

$$
\begin{aligned}
& p=\frac{\rho((C \tilde{u}, B \tilde{v})+(B \tilde{v}, C \tilde{u}))-\left(A_{\rho} \tilde{u}, \tilde{u}\right)-\left(A_{2, \rho} \tilde{v}, \tilde{v}\right)+\omega(C \tilde{u}+B \tilde{v}, C \tilde{u}+B \tilde{v})}{\left(A_{\rho} \tilde{u}, \tilde{u}\right)+\left(A_{2, \rho} \tilde{v}, \tilde{v}\right)}, \\
& q=\frac{-\rho((C \tilde{u}, B \tilde{v})+(B \tilde{v}, C \tilde{u}))}{\left(A_{\rho} \tilde{u}, \tilde{u}\right)+\left(A_{2, \rho} \tilde{v}, \tilde{v}\right)} .
\end{aligned}
$$

Since $A_{\rho}$ and $A_{2, \rho}$ are SPD, and

$$
(C \tilde{u}, B \tilde{v})=\overline{\tilde{u}}^{T} C^{T} B \tilde{v}=\overline{\tilde{u}^{T} C^{T} B \overline{\tilde{v}}}=\overline{\overline{\tilde{v}} B^{T} C \tilde{u}}=\overline{(B \tilde{v}, C \tilde{u})},
$$


we have that $(C \tilde{u}, B \tilde{v})+(B \tilde{v}, C \tilde{u}),\left(A_{\rho} \tilde{u}, \tilde{u}\right)$ and $\left(A_{2, \rho} \tilde{v}, \tilde{v}\right)$ are real numbers, and therefore both $p$ and $q$ are also real numbers. Moreover, we have $\left(A_{\rho} \tilde{u}, \tilde{u}\right)+\left(A_{2, \rho} \tilde{v}, \tilde{v}\right)>0$, since $A_{\rho}$ and $A_{2, \rho}$ are symmetric positive definite and $\left(\tilde{u}^{T}, \tilde{v}^{T}\right)^{T} \neq 0$.

Since $C \tilde{u}+B \tilde{v} \neq 0$, we have

$$
(C \tilde{u}+B \tilde{v}, C \tilde{u}+B \tilde{v})>0 .
$$

Then it follows that

$$
\begin{aligned}
1-q & =\frac{\left(A_{\rho} \tilde{u}, \tilde{u}\right)+\left(A_{2, \rho} \tilde{v}, \tilde{v}\right)+\rho((C \tilde{u}, B \tilde{v})+(B \tilde{v}, C \tilde{u}))}{\left(A_{\rho} \tilde{u}, \tilde{u}\right)+\left(A_{2, \rho} \tilde{v}, \tilde{v}\right)} \\
& =\frac{(A \tilde{u}, \tilde{u})+\left(A_{2} \tilde{v}, \tilde{v}\right)+\rho(C \tilde{u}+B \tilde{v}, C \tilde{u}+B \tilde{v})}{\left(A_{\rho} \tilde{u}, \tilde{u}\right)+\left(A_{2, \rho} \tilde{v}, \tilde{v}\right)} \\
& >0 .
\end{aligned}
$$

If $p \geq 0$, i.e.,

$$
-\rho(C \tilde{u}-B \tilde{v}, C \tilde{u}-B \tilde{v})+\omega(C \tilde{u}+B \tilde{v}, C \tilde{u}+B \tilde{v}) \geq(A \tilde{u}, \tilde{u})+\left(A_{2} \tilde{v}, \tilde{v}\right),
$$

then we have

$$
1+|p|+q=1+p+q=\frac{\omega(C \tilde{u}+B \tilde{v}, C \tilde{u}+B \tilde{v})}{\left(A_{\rho} \tilde{u}, \tilde{u}\right)+\left(A_{2, \rho} \tilde{v}, \tilde{v}\right)}>0
$$

Otherwise, in the cases of $p<0$, i.e.,

$$
-\rho(C \tilde{u}-B \tilde{v}, C \tilde{u}-B \tilde{v})+\omega(C \tilde{u}+B \tilde{v}, C \tilde{u}+B \tilde{v})<(A \tilde{u}, \tilde{u})+\left(A_{2} \tilde{v}, \tilde{v}\right),
$$

then,

$$
\begin{aligned}
1+|p|+q & =1-p+q \\
& =\frac{-2 \rho((C \tilde{u}, B \tilde{v})+(B \tilde{v}, C \tilde{u}))+2\left(A_{\rho} \tilde{u}, \tilde{u}\right)+2\left(A_{2, \rho} \tilde{v}, \tilde{v}\right)-\omega(C \tilde{u}+B \tilde{v}, C \tilde{u}+B \tilde{v})}{\left(A_{\rho} \tilde{u}, \tilde{u}\right)+\left(A_{2, \rho} \tilde{v}, \tilde{v}\right)} \\
& =\frac{2 \rho(C \tilde{u}-B \tilde{v}, C \tilde{u}-B \tilde{v})+2(A \tilde{u}, \tilde{u})+2\left(A_{2} \tilde{v}, \tilde{v}\right)-\omega(C \tilde{u}+B \tilde{v}, C \tilde{u}+B \tilde{v})}{\left(A_{\rho} \tilde{u}, \tilde{u}\right)+\left(A_{2, \rho} \tilde{v}, \tilde{v}\right)} \\
& >\frac{\omega(C \tilde{u}+B \tilde{v}, C \tilde{u}+B \tilde{v})}{\left(A_{\rho} \tilde{u}, \tilde{u}\right)+\left(A_{2, \rho} \tilde{v}, \tilde{v}\right)} \\
& >0 .
\end{aligned}
$$

Therefore, the desired result is obtained by Lemma 2.3.

Lemma 2.5. Assume that $\mu$ is an eigenvalue of $M$ with corresponding eigenvector $\left(\tilde{u}^{T}, \tilde{v}^{T}, \tilde{\lambda}^{T}\right)^{T}$. If $C \tilde{u}+B \tilde{v}=0$, then $|\mu|<1$. 
Proof. Since $C \tilde{u}+B \tilde{v}=0$, it follows from (2.28) and Lemma 2.1 that

$$
-\rho((C \tilde{u}, B \tilde{v})+(B \tilde{v}, C \tilde{u}))=\mu\left(\left(A_{\rho} \tilde{u}, \tilde{u}\right)+\left(A_{2, \rho} \tilde{v}, \tilde{v}\right)\right)
$$

or

$$
|\mu|=\frac{\rho|((C \tilde{u}, B \tilde{v})+(B \tilde{v}, C \tilde{u}))|}{(A \tilde{u}, \tilde{u})+\left(A_{2} \tilde{v}, \tilde{v}\right)+\rho(C \tilde{u}, C \tilde{u})+\rho(B \tilde{v}, B \tilde{v})} .
$$

Further, since $B$ is invertible, we have

$$
\tilde{v}=-B^{-1} C \tilde{u} .
$$

Then, if $\tilde{u}=0$, we have $\tilde{v}=0$, which contradicts with Lemma 2.2. Thus, we have $\tilde{u} \neq 0$, and

$$
(A \tilde{u}, \tilde{u})>0 .
$$

Thus,

$$
\begin{aligned}
& (A \tilde{u}, \tilde{u})+\left(A_{2} \tilde{v} \tilde{v}\right)+\rho(C \tilde{u}, C \tilde{u})+\rho(B \tilde{v}, B \tilde{v})-\rho|((C \tilde{u}, B \tilde{v})+(B \tilde{v}, C \tilde{u}))| \\
= & (A \tilde{u}, \tilde{u})+\left(A_{2} \tilde{v}, \tilde{v}\right)+\rho(C \tilde{u} \pm B \tilde{v}, C \tilde{u} \pm B \tilde{v}) \\
> & 0
\end{aligned}
$$

which implies $|\mu|<1$, the proof is thus completed.

Combining Lemma 2.4 and Lemma 2.5, we obtain the convergence result of the developed augmented Lagrangian Uzawa method as stated in the following theorem.

Theorem 2.1. For any two positive real numbers $\rho$ and $\omega$, the augmented Lagrangian Uzawa method for solving the double saddle-point system (1.1) is convergent.

Remark 2.1. If $A_{2}$ is SPD, then the system (1.1) can be viewed as a standard saddle-point system, and the convergence of the corresponding augmented Lagrangian Uzawa solver is proved in [3] and [10].

Remark 2.2. The positive numbers $\rho$ and $\omega$ is an important factor on the convergence rates, and their optimal values will numerical studied in the next section.

\section{Application to the double saddle-point system arising from DLM/FD finite element method}

In this section, we consider an application of the developed augmented Lagrangian Uzawa method for solving the double saddle-point system (1.1), which arises from the DLM/FD 


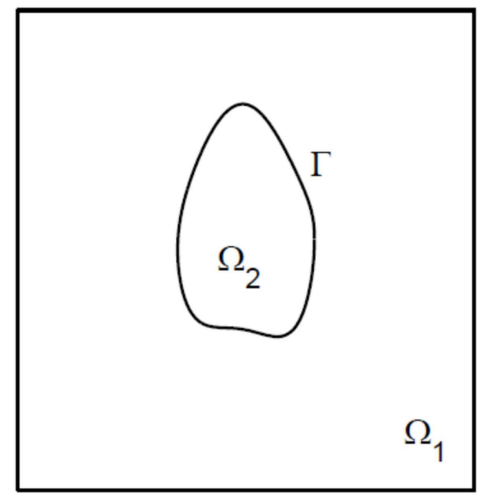

Figure 1: Graphical depiction of the domain with an immersed interface.

finite element method for solving the following elliptic interface problem with discontinuous coefficients:

$$
\begin{aligned}
-\nabla \cdot\left(\beta_{1} \nabla u_{1}\right) & =f_{1}, & & \text { in } \Omega_{1}, \\
-\nabla \cdot\left(\beta_{2} \nabla u_{2}\right) & =f_{2}, & & \text { in } \Omega_{2,} \\
u_{1} & =u_{2}, & & \text { on } \Gamma, \\
\beta_{1} \nabla u_{1} \cdot \boldsymbol{n}_{1}+\beta_{2} \nabla u_{2} \cdot \boldsymbol{n}_{2} & =w, & & \text { on } \Gamma, \\
u_{1} & =0, & & \text { on } \partial \Omega_{1} \backslash \Gamma,
\end{aligned}
$$

where and thereafter, $f_{1} \in L^{2}\left(\Omega_{1}\right), f_{2} \in L^{2}\left(\Omega_{2}\right), w \in H^{1 / 2}(\Gamma), \beta_{1}<\beta_{2}, \Omega=\Omega_{1} \cup \Omega_{2} \subset \mathbb{R}^{d}$ as shown in Fig. 1 , the immersed interface $\Gamma=\partial \Omega_{2}$ is generally a closed curve that divides the domain $\Omega$ into an interior region $\Omega_{2}$ and an exterior region $\Omega_{1}, n_{1}$ and $n_{2}$ stand for the unit outward normal vectors on $\partial \Omega_{1}$ and $\partial \Omega_{2}$, respectively.

We remark that there is another type of immersed $\Omega_{2}$, where $\partial \Omega_{1} \cap \partial \Omega_{2} \neq \varnothing$ and thus the boundary condition of $u_{2}$ (e.g. $u_{2}=0$ ) should be imposed on $\partial \Omega_{1} \cap \partial \Omega_{2}$. In such cases, the Uzawa type iterative method for solving double saddle-point systems arising from the DLM/FD finite element method was proved in the literatures since $A_{2}$ in (1.1) is SPD.

\subsection{Introduction of DLM/FD finite element method to the elliptic interface problem}

Define

$$
\boldsymbol{V}=H_{0}^{1}(\Omega), \quad \boldsymbol{V}_{2}=H^{1}\left(\Omega_{2}\right), \quad \boldsymbol{\Lambda}=\left(\boldsymbol{V}_{2}\right)^{*},
$$

where $\left(V_{2}\right)^{*}$ denotes the dual space of $V_{2}$. Let $T_{h}(\Omega)$ and $T_{H}\left(\Omega_{2}\right)$ be the partitions of $\Omega$ and $\Omega_{2}$, respectively. Denote by $V_{h}$ and $V_{2, H}$ the conforming $P_{1}$ finite element spaces of $\boldsymbol{V}$ and $\boldsymbol{V}_{2}$, respectively. Define $\boldsymbol{\Lambda}_{H}=\boldsymbol{V}_{2, H}$. Let $(\cdot, \cdot)_{D}$ be the $L^{2}$ inner product over $D$, and $\langle\cdot, \cdot\rangle_{D}$ be the $H^{1}$ inner product over $D$. 
The DLM/FD finite element method for solving the elliptic interface problem (3.1)(3.5) is defined as [1]: find $\left(\tilde{u}_{h}, u_{2, H}, \lambda_{H}\right) \in V_{h} \times V_{2, H} \times \Lambda_{H}$, such that

$$
\begin{aligned}
& \left(\tilde{\beta} \nabla \tilde{u}_{h}, \nabla v_{h}\right)_{\Omega}+\left\langle\lambda_{H},\left.v_{h}\right|_{\Omega_{2}}\right\rangle_{\Omega_{2}}=\left(\tilde{f}, v_{h}\right)_{\Omega^{\prime}} \\
& \left(\left(\beta_{2}-\tilde{\beta}\right) \nabla u_{2, H}, \nabla v_{2, H}\right)_{\Omega_{2}}-\left\langle\lambda_{H}, v_{2, H}\right\rangle_{\Omega_{2}}=\left(f_{2}-\tilde{f}_{2}, v_{2, H}\right)_{\Omega_{2}}+\left(w, v_{2, H}\right)_{\Gamma}, \\
& \left\langle\xi_{H},\left.\tilde{u}_{h}\right|_{\Omega_{2}}-u_{2, H}\right\rangle_{\Omega_{2}}=0,
\end{aligned}
$$

where $\tilde{\beta}$ (resp. $\tilde{f}$ ) is an extension of $\beta_{1}$ (resp. $f_{1}$ ) from $\Omega_{1}$ to $\Omega$, and $\tilde{f}_{2}$ is the restriction of $\tilde{f}$ in $\Omega_{2}$.

Let $\left\{\phi_{i}\right\}_{i=1}^{n}$ and $\left\{\psi_{j}\right\}_{j=1}^{m}$ be the nodal basis of $\boldsymbol{V}_{h}$ and $\boldsymbol{V}_{2, H}=\boldsymbol{\Lambda}_{H}$, respectively. Here, $n$ denotes the number of inner nodes in $T_{h}(\Omega)$, and $m$ denotes the number of nodes in $T_{H}(\Omega)$. Then the linear system generated by using the above DLM/FD finite element method is of the form

$$
\left(\begin{array}{ccc}
A & 0 & C^{T} \\
0 & A_{2} & B^{T} \\
C & B & 0
\end{array}\right)\left(\begin{array}{c}
u \\
u_{2} \\
\lambda
\end{array}\right)=\left(\begin{array}{c}
F \\
G \\
0
\end{array}\right)
$$

where the block matrices $A=\left(a_{i j}\right)_{n \times n^{\prime}} A_{2}=\left(\hat{a}_{i j}\right)_{m \times m^{\prime}} B=\left(b_{i j}\right)_{m \times m}$ and $C=\left(c_{i j}\right)_{m \times n}$ are defined as

$$
\begin{array}{ll}
a_{i j}=\left(\tilde{\beta} \nabla \phi_{i}, \nabla \phi_{j}\right)_{\Omega^{\prime}} & \hat{a}_{i j}=\left(\left(\beta_{2}-\tilde{\beta}\right) \nabla \psi_{i}, \nabla \psi_{j}\right)_{\Omega_{2}}, \\
b_{i j}=-\left\langle\psi_{i}, \psi_{j}\right\rangle_{\Omega_{2}}, & c_{i j}=\left\langle\psi_{i}, \phi_{j}\right\rangle_{\Omega_{2}},
\end{array}
$$

and the vectors $F=\left(f_{j}\right)_{n \times 1}$ and $G=\left(g_{j}\right)_{m \times 1}$ are defined by

$$
f_{j}=\left(\tilde{f}, \phi_{j}\right)_{\Omega^{\prime}} \quad g_{j}=\left(f_{2}-\tilde{f}_{2}, \psi_{j}\right)_{\Omega_{2}}+\left(w, \psi_{j}\right)_{\Gamma} .
$$

The well-posedness of the DLM/FD finite element discretization (3.7)-(3.9) was proved by using the ellipticity on the discrete kernel and the discrete inf-sup conditions, see Proposition 3.4 and Proposition 3.5 in [7]. Thus, by the convergence analysis given in Section 2, we can conclude that the developed augmented Lagrangian Uzawa method for solving the double saddle-point problem (3.10) is convergent.

We remark that the convergence rate of the proposed Uzawa type iterative method and the optimal parameters $\rho$ and $\omega$ are not analyzed for solving (3.10) in this paper, since it requires more information about the properties of the sub-matrices $B$ and $C$ that have not been studied for the DLM/FD finite element method on solving any type of interface problem yet. Instead, we carried out some numerical studies in Section 3.2 about the convergence performance of the developed iterative method in terms of different choices of mesh sizes and of parameters $\rho$ and $\omega$. 


\subsection{Numerical experiments}

\subsubsection{Example 1 (the case of smooth interface)}

In this example, based on the meshes $T_{h}(\Omega)$ and $T_{H}\left(\Omega_{2}\right)$ as depicted in Fig. 2, we adopt the DLM/FD finite element method to discretize the elliptic interface problem (3.1)-(3.5) with different $\beta_{1}$ and $\beta_{2}$ and defined in $\Omega=(0,1) \times(0,1)$ with an immersed interface $\Gamma:(x-0.3)^{2}+(y-0.3)^{2}=0.01$. And, the right hand side functions, $f_{1}$ and $f_{2}$, and the jump flux, $\omega$, are appropriately chosen to such that the following function

$$
u(x, y)=\sin (\pi x) \sin (\pi y)\left((x-0.3)^{2}+(y-0.3)^{2}-0.01\right)^{2}
$$

is the exact solution to (3.1)-(3.5).

Then, we use the developed augmented Lagrangian Uzawa method, as shown in Algorithm 1, to solve the double saddle-point problem generated from the DLM/FD finite element discretization, where the stop criteria is

$$
\begin{aligned}
& \left\|u^{(n+1)}-u^{(n)}\right\| \leq \operatorname{Tol} \times\left\|u^{(n+1)}\right\|, \\
& \left\|u_{2}^{(n+1)}-u_{2}^{(n)}\right\| \leq \operatorname{Tol} \times\left\|u_{2}^{(n+1)}\right\|, \\
& \left\|\lambda^{(n+1)}-\lambda^{(n)}\right\| \leq \operatorname{Tol} \times\left\|\lambda^{(n+1)}\right\|,
\end{aligned}
$$

with $T o l=10^{-7}$. Here $\|\cdot\|$ denotes the $l^{2}$-norm of a vector.

We will consider the following four cases:

CASE I: $\beta_{1}=1$ and $\beta_{2}=100$.

CASE II: $\beta_{1}=1$ and $\beta_{2}=1000$.

CASE III: $\beta_{1}=1$ and $\beta_{2}=10000$.

CASE IV: $\beta_{1}=100$ and $\beta_{2}=10000$.

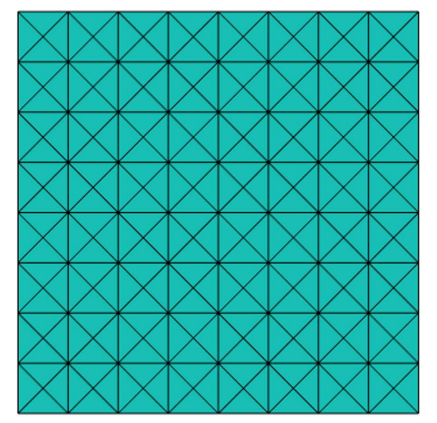

Figure 2: The meshes $T_{h}(\Omega)$ and $T_{H}\left(\Omega_{2}\right)$ in Example 1 . 
Table 1: Results of ALUM for CASE I.

\begin{tabular}{||c|c|c|c|c|c|c|c||}
\hline & & \multicolumn{7}{|c||}{ \# of iteration } \\
\hline$h$ & $H$ & $\rho=\omega=20$ & $\rho=\omega=50$ & $\rho=\omega=100$ & $\rho=\omega=200$ & $\rho=\omega=500$ & $\left\|u-u^{\text {approx }}\right\|_{H^{1}(\Omega)}$ \\
\hline $1 / 8$ & $1 / 16$ & 66 & 45 & 66 & 98 & 210 & $2.8811 \mathrm{e}-02$ \\
\hline $1 / 16$ & $1 / 32$ & 64 & 41 & 59 & 97 & 196 & $1.5141 \mathrm{e}-02$ \\
\hline $1 / 32$ & $1 / 64$ & 76 & 45 & 65 & 93 & 149 & $7.6614 \mathrm{e}-03$ \\
\hline $1 / 64$ & $1 / 128$ & 90 & 44 & 54 & 78 & 139 & $3.8421 \mathrm{e}-03$ \\
\hline $1 / 128$ & $1 / 256$ & 228 & 104 & 60 & 71 & 149 & $1.9225 \mathrm{e}-03$ \\
\hline
\end{tabular}

Table 2: Results of ALUM for CASE II.

\begin{tabular}{||c|c|c|c|c|c|c|c||}
\hline & & \multicolumn{5}{|c||}{ \# of iteration } & \\
\hline$h$ & $H$ & $\rho=\omega=200$ & $\rho=\omega=500$ & $\rho=\omega=1000$ & $\rho=\omega=2000$ & $\rho=\omega=5000$ & $\left\|u-u^{\text {approx }}\right\|_{H^{1}(\Omega)}$ \\
\hline $1 / 8$ & $1 / 16$ & 53 & 40 & 58 & 80 & 174 & $3.0264 \mathrm{e}-02$ \\
\hline $1 / 16$ & $1 / 32$ & 55 & 34 & 53 & 76 & 167 & $1.5396 \mathrm{e}-02$ \\
\hline $1 / 32$ & $1 / 64$ & 67 & 38 & 60 & 81 & 142 & $7.6956 \mathrm{e}-03$ \\
\hline $1 / 64$ & $1 / 128$ & 64 & 35 & 51 & 69 & 141 & $3.8466 \mathrm{e}-03$ \\
\hline $1 / 128$ & $1 / 256$ & 60 & 32 & 46 & 71 & 147 & $1.9230 \mathrm{e}-03$ \\
\hline
\end{tabular}

Table 3: Results of ALUM for CASE III.

\begin{tabular}{||c|c|c|c|c|c|c|c||}
\hline & & \multicolumn{5}{|c||}{ \# of iteration } & \\
\hline$h$ & $H$ & $\rho=\omega=1000$ & $\rho=\omega=5000$ & $\rho=\omega=10000$ & $\rho=\omega=15000$ & $\rho=\omega=20000$ & $\left\|u-u^{\text {approx }}\right\|_{H^{1}(\Omega)}$ \\
\hline $1 / 8$ & $1 / 16$ & 79 & 35 & 47 & 53 & 76 & $9.4813 \mathrm{e}-02$ \\
\hline $1 / 16$ & $1 / 32$ & 84 & 34 & 44 & 60 & 77 & $3.1775 \mathrm{e}-02$ \\
\hline $1 / 32$ & $1 / 64$ & 100 & 36 & 49 & 58 & 68 & $1.0564 \mathrm{e}-02$ \\
\hline $1 / 64$ & $1 / 128$ & 105 & 30 & 44 & 56 & 69 & $4.2710 \mathrm{e}-03$ \\
\hline $1 / 128$ & $1 / 256$ & 106 & 30 & 45 & 58 & 72 & $1.9800 \mathrm{e}-03$ \\
\hline
\end{tabular}

The numerical results of these cases are reported in Tables 1-4 respectively, where the numerical approximation obtained by the augmented Lagrangian Uzawa method at the last step is denoted by $u^{a p p r o x}$. From these tables, we observe that for all fixed parameters $\rho$ and $\omega$ in our numerical experiments, the augmented Lagrangian Uzawa methods are convergent, which confirms the convergence analysis presented in Section 2.2. Moreover, for the elliptic interface problem (3.1)-(3.5), we can see that an simple and good choice of the parameters for the proposed iterative method is $\rho=\omega=\max \left\{\beta_{1}, \beta_{2}\right\}$, although it is not the optimal parameters in some cases with a fixed mesh size.

Next, we numerically study the convergence rate of the proposed iterative algorithm. To that end, we report the semi-log plots of the error, $\ln \left(\left\|u-u^{a p p r o x}\right\|_{H^{1}(\Omega)}\right)$, against the iteration step for four cases with different mesh sizes in Fig. 3, and for Case IV with different parameters $\rho$ and $\omega$ in Fig. 4. Fig. 3 illustrates that the proposed iterative algorithm 
Table 4: Results of ALUM for CASE IV.

\begin{tabular}{||c|c|c|c|c|c|c|c||}
\hline & & \multicolumn{5}{|c||}{ \# of iteration } & \\
\hline$h$ & $H$ & $\rho=\omega=1000$ & $\rho=\omega=5000$ & $\rho=\omega=10000$ & $\rho=\omega=15000$ & $\rho=\omega=20000$ & $\left\|u-u^{\text {approx }}\right\|_{H^{1}(\Omega)}$ \\
\hline $1 / 8$ & $1 / 16$ & 120 & 45 & 67 & 72 & 98 & $2.8811 \mathrm{e}-02$ \\
\hline $1 / 16$ & $1 / 32$ & 118 & 41 & 59 & 62 & 96 & $1.5141 \mathrm{e}-02$ \\
\hline $1 / 32$ & $1 / 64$ & 138 & 45 & 65 & 81 & 93 & $7.6614 \mathrm{e}-03$ \\
\hline $1 / 64$ & $1 / 128$ & 160 & 44 & 55 & 66 & 77 & $3.8421 \mathrm{e}-03$ \\
\hline $1 / 128$ & $1 / 256$ & 411 & 104 & 60 & 58 & 70 & $1.9225 \mathrm{e}-03$ \\
\hline
\end{tabular}
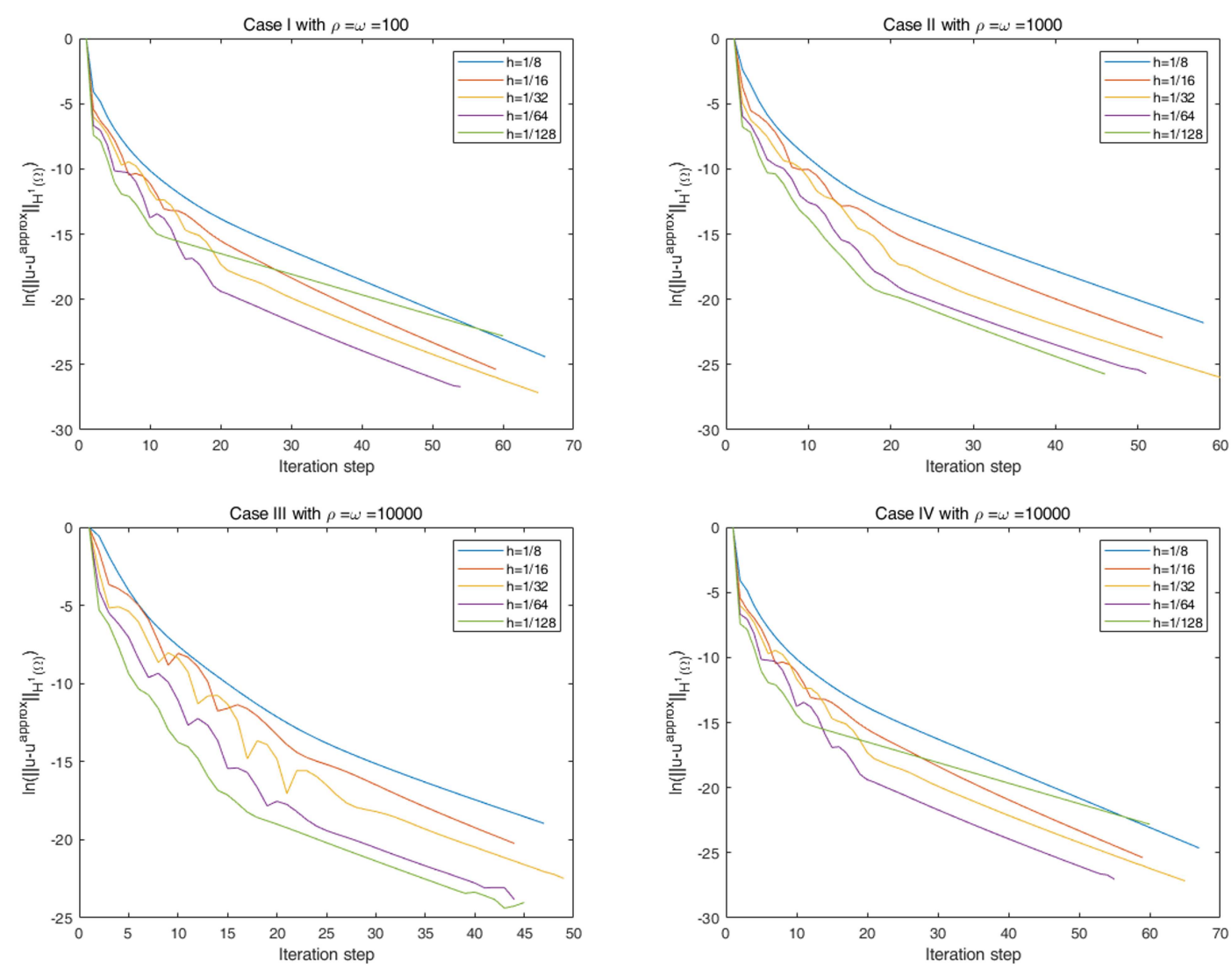

Figure 3: Convergence rates of the proposed method for Example 1 with different mesh sizes.

converges almost exponentially for all mesh sizes in all cases after certain amount of iterative steps. Fig. 4 displays that the convergence rate of the proposed method depends on the choices of parameters $\rho$ and $\omega$. 


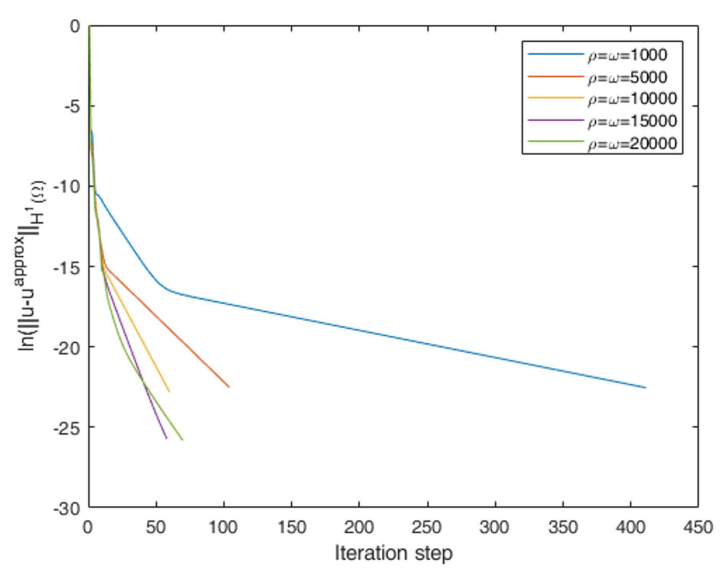

Figure 4: Convergence rates of the proposed method for Case IV with different $\rho$ and $\omega$.

\subsubsection{Example 2 (the case of piecewisely smooth interface)}

In this example, we study the convergence rate of the proposed method when the interface is not smooth. Let $\Omega=(0,1) \times(0,1)$ and $\Omega_{2}$ is 5-pointed star with vertices

$$
X_{i}=0.25 r_{i} \cos \left(\frac{\pi i}{5}+t_{0}\right)+0.375, \quad Y_{i}=0.25 r_{i} \sin \left(\frac{\pi i}{4}+t_{0}\right)+0.375, \quad i=1, \cdots, 10,
$$

where $t_{0}=0.1243, r_{i}=0.35+0.3(i \bmod 2)$. The meshes $T_{h}(\Omega)$ and $T_{H}\left(\Omega_{2}\right)$ are depicted in Fig. 5.

The coefficients $\beta_{i}(i=1,2)$ defined as

CASE V: $\beta_{1}=1$ and $\beta_{2}=100$.

CASE VI: $\beta_{1}=1$ and $\beta_{2}=1000$.
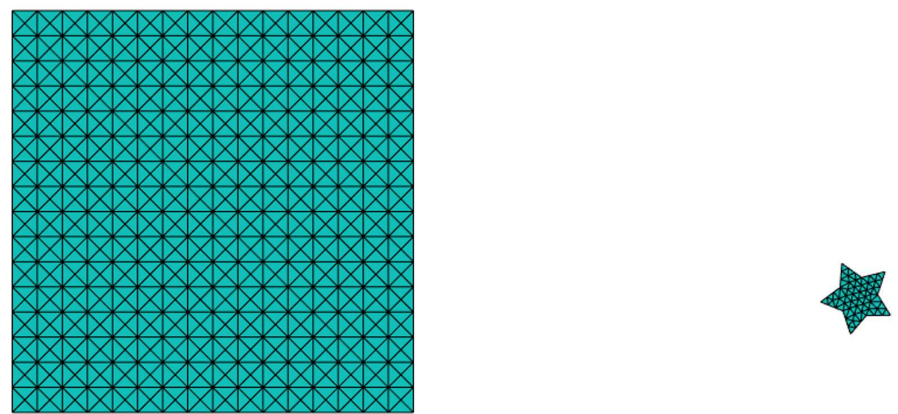

Figure 5: The meshes $T_{h}(\Omega)$ and $T_{H}\left(\Omega_{2}\right)$ in Example 2. 
Table 5: Results of ALUM for Example 2.

\begin{tabular}{|c|c|c|c|c|}
\hline$h$ & $H$ & \# of iteration & $\left\|u-u^{\text {approx }}\right\|_{H^{1}(\Omega)}$ & \\
\hline $1 / 8$ & $1 / 16$ & 109 & $5.0921 \mathrm{e}-01$ & \multirow{5}{*}{$\begin{array}{l}\text { Case V with } \\
\rho=\omega=150\end{array}$} \\
\hline $1 / 16$ & $1 / 32$ & 121 & $2.1087 \mathrm{e}-01$ & \\
\hline $1 / 32$ & $1 / 64$ & 103 & $1.0565 \mathrm{e}-01$ & \\
\hline $1 / 64$ & $1 / 128$ & 107 & $5.1449 \mathrm{e}-02$ & \\
\hline $1 / 128$ & $1 / 256$ & 141 & $2.5586 \mathrm{e}-02$ & \\
\hline $1 / 8$ & $1 / 16$ & 78 & $7.5383 \mathrm{e}-01$ & \multirow{5}{*}{$\begin{array}{c}\text { Case VI } \\
\rho=\omega=1000\end{array}$} \\
\hline $1 / 16$ & $1 / 32$ & 91 & $2.9976 \mathrm{e}-01$ & \\
\hline $1 / 32$ & $1 / 64$ & 83 & $1.3740 \mathrm{e}-01$ & \\
\hline $1 / 64$ & $1 / 128$ & 83 & $5.5993 \mathrm{e}-02$ & \\
\hline $1 / 128$ & $1 / 256$ & 81 & $2.7089 \mathrm{e}-02$ & \\
\hline $1 / 8$ & $1 / 16$ & 80 & $1.7814 \mathrm{e}+00$ & \multirow{5}{*}{$\begin{array}{c}\text { Case VII } \\
\rho=\omega=10000\end{array}$} \\
\hline $1 / 16$ & $1 / 32$ & 94 & $5.2502 \mathrm{e}-01$ & \\
\hline $1 / 32$ & $1 / 64$ & 86 & $2.6354 \mathrm{e}-01$ & \\
\hline $1 / 64$ & $1 / 128$ & 88 & 7.9495e-02 & \\
\hline $1 / 128$ & $1 / 256$ & 85 & $3.4198 \mathrm{e}-02$ & \\
\hline $1 / 8$ & $1 / 16$ & 78 & $7.5383 e-01$ & \multirow{5}{*}{$\begin{array}{c}\text { Case VIII } \\
\rho=\omega=10000\end{array}$} \\
\hline $1 / 16$ & $1 / 32$ & 91 & $2.9976 \mathrm{e}-01$ & \\
\hline $1 / 32$ & $1 / 64$ & 83 & $1.3740 \mathrm{e}-01$ & \\
\hline $1 / 64$ & $1 / 128$ & 83 & $5.5993 \mathrm{e}-02$ & \\
\hline $1 / 128$ & $1 / 256$ & 81 & $2.7089 \mathrm{e}-02$ & \\
\hline
\end{tabular}

CASE VII: $\beta_{1}=1$ and $\beta_{2}=10000$.

CASE VIII: $\beta_{1}=10$ and $\beta_{2}=10000$.

will be used in the following numerical studies. The coefficients in the elliptic interface problem (3.1)-(3.5) are chosen to satisfy that the following function

$$
u(x, y)=\sin (2 \pi x) \sin (2 \pi y)
$$

is the exact solution to (3.1)-(3.5).

Numerical results of this example are reported in Table 5, and convergence performances of the developed iterative algorithm are illustrated in Figs. 6 and 7. From them we can observe the similar numerical phenomena as shown in Example 1. We note that the numerical oscillation is a little more significant in this example when the stopping criterion is nearly reached, which may be possibly caused by the bit depth of the machine's floating point operation. 

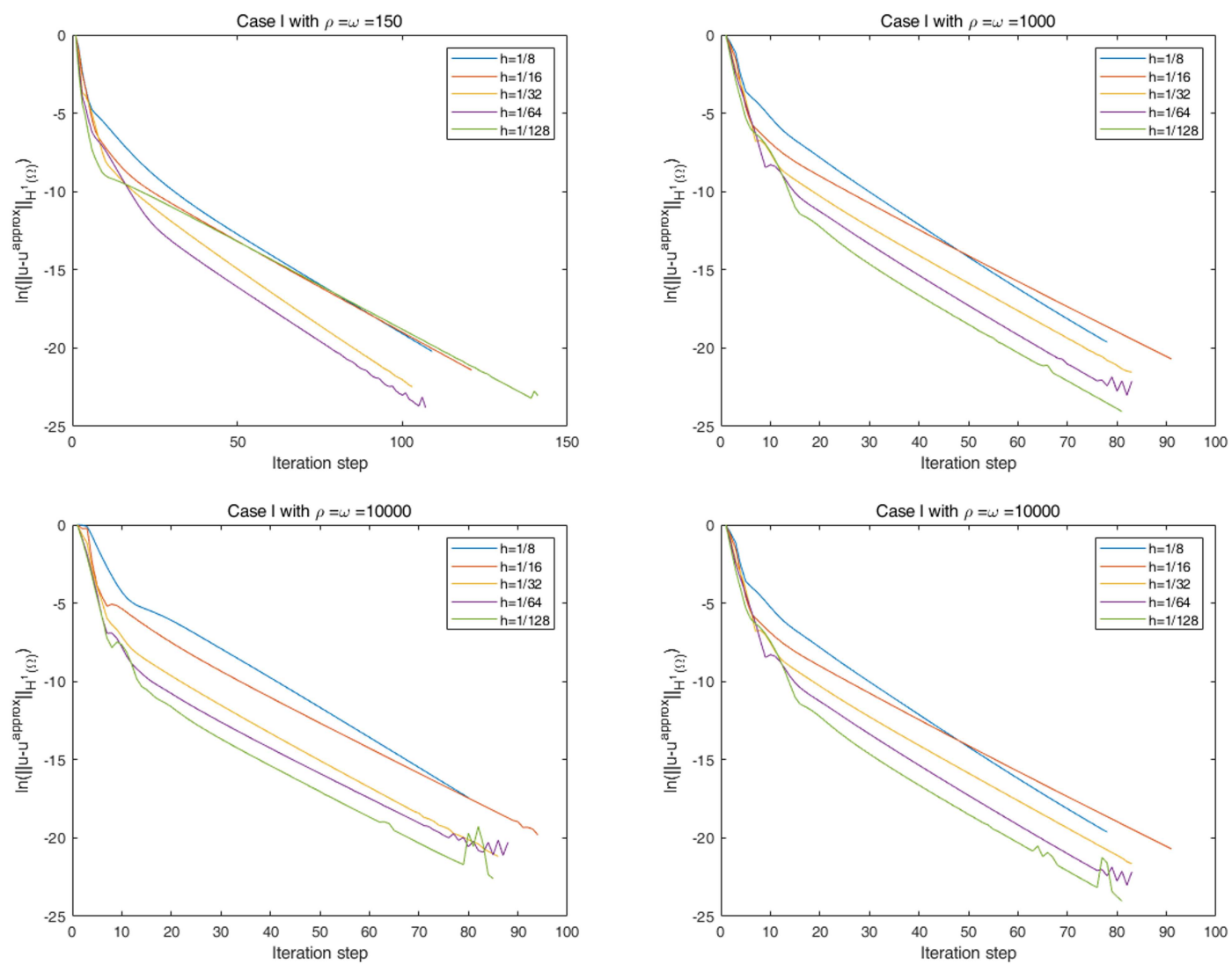

Figure 6: Convergence rates of the proposed method for Example 2 with different mesh sizes.

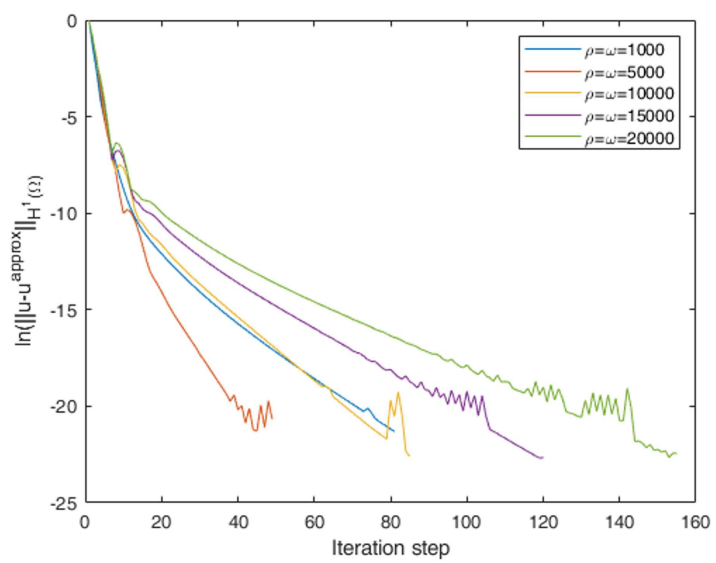

Figure 7: Convergence rates of the proposed method for Case VIII with different $\rho$ and $\omega$. 


\section{Conclusion and future work}

The coefficient matrix of a double saddle-point problem has more complicated structure than that of a standard saddle-point problem. Since the sub-block $A_{2}$ in (1.2) is symmetric positive semidefinite, the convergence of the augmented Lagrangian Uzawa method for solving the double saddle-point problem (1.1) cannot be guaranteed by the existing convergence results proved for a standard saddle-point problem. The analysis and numerical experiments in this paper show that the augmented Lagrangian Uzawa method is an effective and convergent solver for the double saddle-point system (1.1), which may arise from the DLM/FD finite element method for elliptic interface problems. The convergence analysis and the optimal parameters for the developed iterative algorithm may be possibly derived from some essential properties of sub-blocks $B$ and $C$, which however have not been studied for the DLM/FD finite element method on solving any kinds of interface problems yet. Though, we can affirm that the presented Uzawa method might be employed to solve more types of large-scale double saddlepoint systems which are generated by the DLM/FD finite element method for Stokes-, Stokes/elliptic-, Stokes/parabolic interface problems, and finally fluid structure interaction problems, which are going to be of our high interest in the future.

\section{Acknowledgments}

P. Sun was supported by NSF Grant DMS-1418806. C. Wang was supported by the 10 plus 10 project of Tongji University (No. 4260141304/004/010).

\section{References}

[1] F. Auricchi, D. Boffi, L. Gastaldi, A. Lefieux, and A. Reali. On a fictitious domain method with distributed Lagrange multiplier for interface problems. Applied Numerical Mathematics, 95(0):36-50, 2015.

[2] G. M. Awanou and M. J. Lai. On convergence rate of the augmented Lagrangian algorithm for nonsymmetric saddle point problems. Applied Numerical Mathematics, 54(2):122134, 2005.

[3] C. Bacuta. A unified approach for Uzawa algorithms. SIAM Journal on Numerical Analysis, 44(6):2633-2649, 2006.

[4] F.P.A. Beik and M. Benzi. Iterative methods for double saddle point systems. SIAM Journal on Matrix Analysis \& Applications, 39(2):902-921, 2018.

[5] M. Benzi and F.P.A. Beik. Uzawa-type and augmented lagrangian methods for double saddle point systems. In Prof. Eugene Tyrtyshnikov Prof. Marc Van Barel Prof. Dario Andrea Bini, Prof. Fabio Di Benedetto, editor, Structured Matrices in Numerical Linear Algebra, chapter 11, Springer International Publishing, 2019.

[6] M. Benzi, G.H. Golub, and J. Liesen. Numerical solution of saddle point problems. Acta Numerica, 14:1-137, 2005. 
[7] D. Boffi, L. Gastaldi, and M. Ruggeri. Mixed formulation for interface problems with distributed Lagrange multiplier. Computers \& Mathematics with Applications, 68(12, Part B):21512166, 2014.

[8] J. Bramble, J. Pasciak, and A. Vassilev. Uzawa type algorithms for nonsymmetric saddle point problems. Mathematics of Computation, 69(230):667-689, 2000.

[9] M. R. Cui. Analysis of iterative algorithms of Uzawa type for saddle point problems. Applied Numerical Mathematics, 50(2):133-146, 2004.

[10] R. Glowinski and P. LeTallec. Augmented Lagrangian and Operator Splitting Methods in Nonlinear Mechanics. SIAM, Philadelphia, PA, 1989.

[11] R. Glowinski, T.-W. Pan, T.I. Hesla, and D.D. Joseph. A distributed Lagrange multiplier / fictitious domain method for particulate flows. International Journal of Multiphase Flow, 25(5):755-794, 1999.

[12] R. Glowinski, T.W. Pan, T.I. Hesla, D.D. Joseph, and J. Periaux. A fictitious domain approach to the direct numerical simulation of incompressible viscous flow past moving rigid bodies: Application to particulate flow. Journal of Computational Physics, 169(2):363-426, 2001.

[13] N. Huang. Variable parameter Uzawa method for solving a class of block three-by-three saddle point problems. Numerical Algorithms, 85:1233-12542020.

[14] D. Jia, Z. Sheng, and G. Yuan. An extremum-preserving iterative procedure for the imperfect interface problem. Communications in Computational Physics, 25(3):853-870, MAR 2019.

[15] Z. Liang and G. Zhang. Alternating positive semidefinite splitting preconditioners for double saddle point problems. Calcolo, 56(3):26, 2019.

[16] A. Lundberg, P. Sun, and C. Wang. Distributed Lagrange multiplier-fictitious domain finite element method for Stokes interface problems. Int. J. Numer. Anal. Model., 16(6):939-963, 2019.

[17] A. Lundberg, P. Sun, C. Wang, and C-S. Zhang. Distributed Lagrange multiplier/fictitious domain finite element method for a transient Stokes interface problem with jump coefficients. Computer Modeling in Engineering \& Sciences, 119(1):35-62, 2019.

[18] K. Mardal, B.F. Nielsen, and M. Nordaas. Robust preconditioners for PDE-constrained optimization with limited observations. Bit Numerical Mathematics, 57:405-431, 2015.

[19] T.S. Martynova. On augmented lagrangian methods for saddle-point linear systems with singular or semidefinite $(1,1)$ blocks. Journal of Computational Mathematics, 032(3):297-305, 2014.

[20] J.J.H Miller. On the location of zeros of certain classes of polynomials with applications to numerical analysis. IMA Journal of Applied Mathematics, 8(3):397-406, 1971.

[21] J. Sogn and W. Zulehner. Schur complement preconditioners for multiple saddle point problems of block tridiagonal form with application to optimization problems. IMA J. Numer. Anal., 39(3):1328-1359, 2019.

[22] P. Sun. Fictitious domain finite element method for Stokes/elliptic interface problems with jump coefficients. Journal of Computational and Applied Mathematics, 356:81-97, 2019.

[23] P. Sun and C. Wang. Distributed Lagrange multiplier/fictitious domain finite element method for Stokes/parabolic interface problems with jump coefficients. Applied Numerical Mathematics, 152:199-220, 2020.

[24] C. Wang and P. Sun. A fictitious domain method with distributed Lagrange multiplier for parabolic problems with moving interfaces. Journal of Scientific Computing, 70(2):1-31, 2016.

[25] N. Wang, J. Li, G. Li, and X. Kong. Variants of the Uzawa method for three-order block saddle point problem. Applied Mathematics \& Computation, 305:188-202, 2017.

[26] Q. Wang, Z. Zhang, and E. Zheng. PPIFE method with non-homogeneous flux jump condi- 
tions and its efficient numerical solver for elliptic optimal control problems with interfaces. Numerical Mathematics-Theory Methods and Applications, 13(3):719-744, AUG 2020.

[27] C. Yang, T. Wang, and X. Xie. An interface-unfitted finite element method for elliptic interface optimal control problems. Numerical Mathematics-Theory Methods and Applications, 12(3):727-749, AUG 2019.

[28] Z. Yu. A DLM/FD method for fluid/flexible-body interactions. Journal of Computational Physics, 207(1):1-27, 2005.

[29] N. Zhang, T.T. Lu, and Y. Wei. Semi-convergence analysis of Uzawa methods for singular saddle point problems. Journal of Computational and Applied Mathematics, 255:334-345, 2014.

[30] B. Zheng, Z-Z. Bai, and X. Yang. On semi-convergence of parameterized Uzawa methods for singular saddle point problems. Linear Algebra \& Its Applications, 431(5):808-817, 2009. 